\title{
Image Retrieval with Relevance Feedback using SVM Active Learning
}

\author{
Truong-Giang Ngo ${ }^{1}$, Quoc-Tao Ngo $^{2}$, and Duc-Dung Nguyen ${ }^{3}$ \\ ${ }^{1}$ Department of Information Technology, HaiPhong Private University \\ ${ }^{2,3}$ Institute of Information Technology, Vietnamese Academy of Sciences and Technology
}

\begin{tabular}{l}
\hline \hline Article Info \\
\hline Article history: \\
Received Jun 26, 2016 \\
Revised Aug 25, 2016 \\
Accepted Sep 8, 2016 \\
\hline
\end{tabular}

\section{Keyword:}

Interactive image retrieval

Content-based image retrieval

Relevance feedback

SVM Active learning

Batch mode active learning

\begin{abstract}
In content-based image retrieval, relevant feedback is studied extensively to narrow the gap between low-level image feature and high-level semantic concept. In general, relevance feedback aims to improve the retrieval performance by learning with user's judgements on the retrieval results. Despite widespread interest, but feedback related technologies are often faced with a few limitations. One of the most obvious limitations is often requiring the user to repeat a number of steps before obtaining the improved search results. This makes the process inefficient and tedious search for the online applications. In this paper, a effective feedback related scheme for contentbased image retrieval is proposed. First, a decision boundary is learned via Support Vector Machine to filter the images in the database. Then, a ranking function for selecting the most informative samples will be calculated by defining a novel criterion that considers both the scores of Support Vector Machine function and similarity metric between the "ideal query" and the images in the database. The experimental results on standard datasets have showed the effectiveness of the proposed method..
\end{abstract}

Copyright (c) 2016 Institute of Advanced Engineering and Science. All rights reserved.

\section{Corresponding Author:}

Ngo Truong Giang

Department of Information Technology, HaiPhong Private University

No.36 Dan Lap Road, Hai Phong, Vietnam

Phone: +84904051206

Email: giangnt@hpu.edu.vn

\section{INTRODUCTION}

The rapid development of digital devices and the dominance of social networks have led to the great demand of sharing, browsing and searching images. Therefore, to satisfy such requirements, image retrieval systems have become an urge necessity. Basically, there are two main frameworks to form image retrieval systems: text-based and content-based systems [1]. In text-based image retrieval systems, the users' queries are composed by key-words, which describe image content. The system retrieves images based on image labels which are annotated manually. However, the difficulties in annotating a massive number of images and avoiding subjectively labelling make this framework impractical. In order to overcome such hindrances, Content-Based Image Retrieval (CBIR) is known to be a more optimized approach which aims to bring image content closer to human understanding.

In CBIR, low-level visual features, such as colors, textures, patterns, and shapes are used to describe image contents. These low-level features are automatically extracted to represent the images in the database without manual interventions. Its advantage over keyword based image retrieval lies in the fact that feature extraction can be performed automatically and the image's own content is always consistent. However, the most challenging problem in the CBIR systems is the semantic gap [2], [3], i.e., images of dissimilar semantic content may share some common low-level features, while images of similar semantic content may be scattered in the feature space. Despite the great deal of research work dedicated to the exploration of an ideal descriptor for image content [4], [5], [6], [7], [8], [9] its performance is far from satisfactory due to the fundamental 
difference between human understanding (high level concepts) and machine understanding (low level features). To narrow down the semantic gap, one possible solution is to integrate human interaction in the system, which is popularly known as Relevance Feedback (RF) [10], [11]. In general, RF aims to improve the retrieval performance by learning with user's judgments on the retrieval results. In this way, the system needs to be run through several iterations. In each iteration, the CBIR system fist returns a short list of top-ranked images with respect to a user's query by a regular retrieval approach based on Euclidean distance measure, and then some images are given to users, labeled by them as being relevant or irrelevant (positive or negative examples). Using these labeled images as seeds, machine learning techniques will be used to build a model to classify the database images into two classes: a class containing images that suppose to satisfy the users and the other class containing the irrelevant images. A typical scenario for a CBIR system with RF using machine learning [2] (represented in Figure 1) is as follows:

1. User chooses the query image. Extracting low-level features of the query image

2. Returning result images. There are two cases:

- Initial phase: depends on the similarity measure of low-level features between query image's features and database image's features since we don't have any training example to train machine learning classification.

- Result images in RF loops: Using the function of the classification as a ranking function.

3. User judges these initial result images as to whether and to what degree, they are relevant (positive examples)/irrelevant (negative examples) to the query example. After judging, these images are labeled.

4. Machine learning algorithm is applied to learn the user feedback using labeled examples obtained from the first to the current iteration. Then go back to Step 2.

Note that in this scenario, Step 2, 3 and 4 are repeated until the user is satisfied with the results.

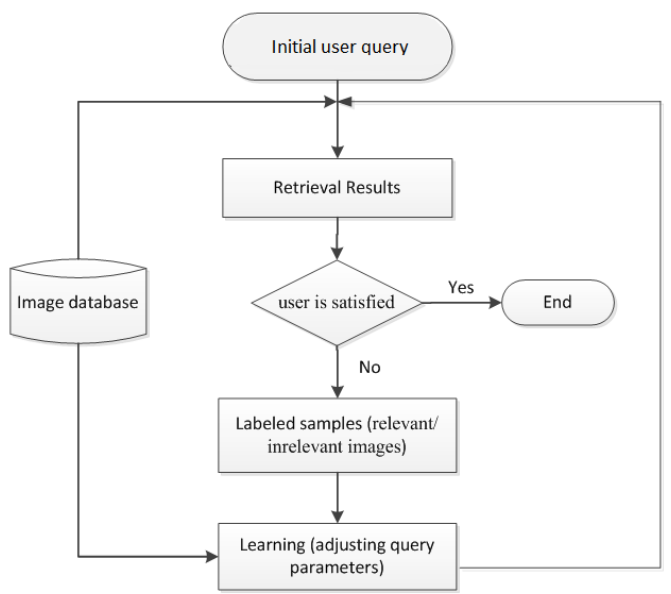

Figure 1. The CBIR system with Relevance Feedback [2]

From a general machine learning view, RF is essentially a binary classification problem in which sample images provided by the user are employed to train a classifier, which is then used to classify the database into images that are relevant to the query and those that are not [1], [2]. However, RF is very different from the traditional classification problem because the feed backs provided by the user are often limited in real-world image retrieval systems. Therefore, small sample learning methods are most promising for RF.

Support Vector Machine (SVM) is one of the popular small sample learning methods widely used in recent years, which has a very good performance for pattern classification problems [12], [13], [14], [15], [16]. Compared with other learning algorithms, SVM appears to be a good candidate for several reasons: generalization ability, without restrictive assumptions regarding the data, fast learning and evaluation for relevance feedback, flexibility, e.g., prior knowledge can be easily used to tune its kernels. However, for the SVM-based 
relevance feedback, the retrieval performance is actually worse when the number of labeled positive feedback samples is small.

SVM active learning actively selects samples close to the boundary as the most informative samples for the user to label in each round of RF [17], [18], [19]. Although SVM active-based relevance feedback can work better than the conventional SVM-based relevance feedback, it has two major drawbacks: First, the performance of SVM is usually limited by the number of labeled examples. Second, since the batch of examples is selected all at once, the previously labeled examples will have no influence on the selection of the rest examples in the batch. To solve this problem, Hoi et al. [20] recently have been proposed the Semi-Supervised SVM Batch Mode Active Learning. This method first constructs a kernel function which is learned from a mixture of labelled and unlabelled examples. The kernel will then be used to effectively identify the informative and diverse examples for active learning via a minmax framework. Zhang et al [21] have been proposed a dynamic batch mode SVM active learning scheme, which dynamically select a batch of examples one by one, using the label of the previously selected example to guide the selection of the next one. The selection of feedback examples is determined by both the existing classification boundary and previously labelled examples. In the solutions presented, the selection of examples for the user to label in each round of RF is solely determined by the existing SVM decision boundary. However, in early iterations, the SVM decision boundary might not be accurate due to the lack of training examples. In this case, the samples selected by the those methods will not be those that should be selected, and it makes the subsequent learning inefficient. Consequently, a poor retrieval performance will result, even if several rounds of learning have been performed.

To address the above problems, we propose a novel Batch Mode for SVM active learning. In proposed method, a decision boundary first is learned via SVM to filter the images in the database. Then, a ranking function will be constructed by defining a novel criterion that considers both the scores of SVM function and similarity measure between the query and the images in the database. This can effectively reduce the adverse effect of inaccurate decision boundary. By using the priority coefficient in the ranking function, We can select a batch of feedback examples which may be informative enough to improve the retrieval accuracy significantly. The experimental results on standard datasets have showed the effectiveness of the proposed method, especially when the number of initially labelled samples is small in early iterations.

The rest of this paper is organized as follows. Section 2 presents the basic theory about SVM-based RF. Section 3 presents the problem formulation and our solution. The retrieval performance of the proposed method is presented in Section 4. Finally, we discuss future research directions and give the conclusions.

\section{SVM-BASED RELEVANCE FEEDBACK}

SVM was first introduced by Vapnik et al. in [22] and until now is an active part of the machine learning research around the world. With strong theoretical foundations available, it is being used for many applications and is a popular small sample learning method that has a very good performance for pattern classification problems. The key idea of SVM is, given a set of n labelled examples $\mathcal{L}=\left\{\left(\mathbf{x}_{1}, y_{1}\right), \ldots,\left(\mathbf{x}_{l}, y_{l}\right)\right\}$, where $\mathbf{x}_{i} \in \mathbb{R}^{d}$ represents an image by a d-dimensional vector, and $y_{i} \in\{1,-1\}$ is the label, to find a hyperplane.

$$
f(x)=(\mathbf{w . x})+b
$$

that achieves the best separation of two classes, provided that the empirical risk is minimized and the margin is maximized for the training vectors that are correctly classified. This is a quadratic programming problem. It is solved by finding $\mathbf{w}$ and $b$ so as to minimize the function

$$
\frac{1}{2}\|\mathbf{w}\|^{2}+C \sum_{i=1}^{n} \xi_{i} \quad \text { s.t. } y_{i}\left(\mathbf{w . x}_{i}+b\right) \geq 1-\xi_{i}, \xi_{i} \geq 0, i=1 \ldots n \text {. }
$$

The corresponding dual form can be the following: Find the parameters $\alpha_{i}, i=1 \ldots n$, which maximize the function

$$
\begin{aligned}
& L(\alpha)=\sum_{i=1}^{n} \alpha_{i}-\frac{1}{2} \sum_{i, j=1}^{n} \alpha_{i} \alpha_{j} y_{i} y_{j} K\left(\mathbf{x}_{i} \cdot \mathbf{x}_{j}\right) \\
& \text { s.t. } \sum_{i=1}^{n} y_{i} \alpha_{i}=0,0 \leqslant \alpha_{i} \leqslant C, i=1 \ldots n,
\end{aligned}
$$


where $K\left(\mathbf{x}_{i} \cdot \mathbf{x}_{j}\right)$ is a kernel function. There are many kernel functions for nonlinear mapping. We choose to use the Gaussian radial basis function as the kernel function in our experiments

$$
K(x, y)=\exp \frac{-(x-y)^{2}}{\sigma^{2}}
$$

where parameter $\sigma$ is the width of the Gaussian function. For a given kernel function, the SVM classifier is given by

$$
f(x)=\operatorname{sign}\left(\sum_{i=1}^{l} \alpha_{i} y_{i} K\left(\mathbf{x}_{i} \cdot \mathbf{x}_{j}\right)+b\right)
$$

and the decision boundary is $\sum_{i=1}^{l} \alpha_{i} y_{i} K\left(\mathbf{x}_{i} \cdot \mathbf{x}_{j}\right)+b=0$.

In SVM-based CBIR relevance feedback, the decision boundary has been used to measure the relevance between a given pattern and the query image. In general, the examples have the large absolute values of SVM functions, the corresponding prediction confidence will be high. In a traditional method for relevance feedback, users judge on the top-ranked image examples, which have the largest values of the SVM function $f(x)$. This strategy is called Passive feedback. It tends to choose the most relevant examples. But they might not be the most informative examples for training SVM. Active learning method is proposed to deal with this problem. Active learning, known as pool-based active learning, is a subfield of machine learning and is one of the most promising methods currently available. Active learning tends to choose the most uncertain examples which are close to the decision boundary of SVM.

\section{BATCH MODE FOR SVM ACTIVE LEARNING}

In CBIR system, the RF can be formulated as an active learning problem, that the most informative unlabeled examples will be selected for improving the classification performance. Let $\mathcal{L}=\left\{\left(x_{1}, y_{1}\right), \ldots,\left(x_{l}, y_{l}\right)\right\}$ denote the labeled image examples that are solicited through $\mathrm{RF}$, and $\mathcal{U}=\left\{x_{l+1}, \ldots, x_{l+u}\right\}$ the unlabeled image examples, where $x_{i} \in \mathbb{R}^{d}$ represents an image by a d-dimensional vector. Let $\mathcal{S}$ be a set of $k$ unlabeled image examples to be selected in $\mathrm{RF}$, and $\operatorname{risk}(f, \mathcal{S}, \mathcal{L}, \mathcal{U})$ be a risk function that depends on the classifier $f$. In [20], selecting the most informative unlabeled examples for the RF is defined as finding the assignment vector $S^{*}$, which minimizes the risk function.

$$
S^{*}=\underset{S \subseteq \mathcal{U} \wedge|S|=k}{\arg \min } \operatorname{risk}(f, \mathcal{S}, \mathcal{L}, \mathcal{U})
$$

The SVM-based active learning method selects the unlabeled example that is closest to the decision boundary. This can be expressed by the following optimization problem

$$
x^{*}=\underset{x \in \mathcal{U}}{\arg \min }|f(x)|
$$

For a query, after the boundary is learned based on the user's feedback, the images in the database are filtered by the decision boundary. However, in early iterations, the SVM decision boundary might not be accurate due to the lack of training examples. Consequently, a poor retrieval performance will result. In this case, similarity measure of low-level features may be more reliable and can be used to restrict this problem. Therefore, we propose a method that can combine two scores of SVM function and similarity measure to form a unique ranking function.

Let $D S_{i}$ denote the distance of the image $i$ from the decision boundary given by SVM active learning, and

$$
\left.D S\left(\mathbf{x}_{i}\right)=\left|f\left(\mathbf{x}_{i}\right)\right|=\mid \mathbf{w} \cdot \mathbf{x}_{i}+b\right) \mid
$$

where $\mathbf{w}$ and $b$ denote the normal vector and the bias of the separating hyperplane, respectively, and $\mathbf{x}_{i}$ is the feature vector representing the image i. Let $D E_{i}$ denote the Euclidean distance obtained between the image $i$ with the "ideal query" image $c$, and

$$
D E\left(\mathbf{x}_{i}\right)= \begin{cases}\left\|\mathbf{x}_{i}-\mathbf{x}_{c}\right\| & \text { if } f\left(\mathbf{x}_{i}\right) \geq 0 \\ \infty & \text { otherwise }\end{cases}
$$


where $\mathbf{x}_{c}=\arg \max _{\mathbf{x}_{j} \in \mathcal{U}} D S\left(x_{j}\right)$. The ranking function of our method for the $i$-th image can be defined as follows.

$$
D S E\left(\mathbf{x}_{i}\right)=\frac{N_{\text {rel }}}{N_{\text {rel }}+N_{\text {nonrel }}} D S\left(\mathbf{x}_{i}\right)+\left(1-\frac{N_{\text {rel }}}{N_{\text {rel }}+N_{\text {nonrel }}}\right) D E\left(\mathbf{x}_{i}\right)
$$

where $N_{r e l}$ is the total number of relevant images and $N_{\text {nonrel }}$ is the total number of non-relevant images in each loop. We will choose the unlabeled examples, which have the smallest values of the ranking function $D S E$ for the user to label.

$$
x^{*}=\underset{x \in \mathcal{U}}{\arg \min } \operatorname{DSE}(x)
$$

The overall algorithm of batch mode for SVM active learning is briefly described in Algorithm 1.

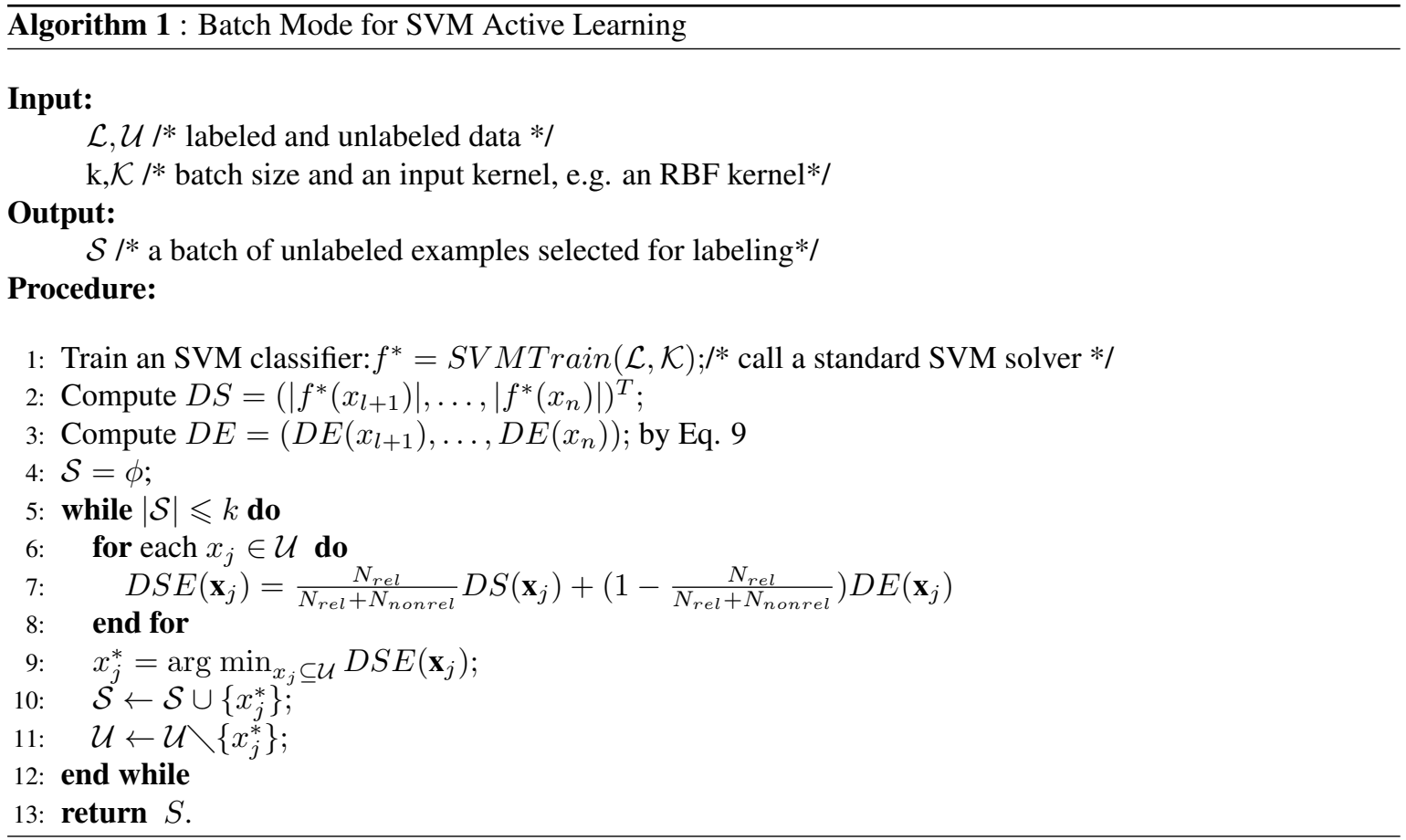

\section{RESULT AND ANALYSIS}

To evaluate the performance of the proposed algorithm, we conduct an extensive set of CBIR experiments by comparing the proposed algorithm to several SVM feedback methods that have been used in image retrieval. The image database is a selected subset from Corel Gallery, which contains 10800 images from about 80 different categories, autumn, aviation, bonsai, castle, cloud, dog, elephant, iceberg, primates, ship, tiger.... Each category consists of about 100 images and all the images are category-homogeneous. For feature representation in the experiment, we extract three types of features: color, texture and shape, which are used in [20].

For color, we selected the color moments. Firstly, we convert the color space from RGB into HSV. Then, we extract 3 moments: color mean, color variance and color skewness in each color channel, respectively. Thus, a 9 -dimensional color moment is used.

For texture, a pyramidal wavelet transform (PWT) is performed on the gray images. Each wavelet decomposition on a gray $2 \mathrm{D}$-image results in four scaled-down subimages. In total, 3 -level decomposition is conducted and features are extracted from 9 of the subimages by computing entropy. Thus, a 9-dimensional wavelet vector is used. Thus, in total, a 36-dimensional feature vector is used to represent each image.

For shape, the edge direction histogram $(\mathrm{EDH})$ is used as the shape features. The edge information contained in the images is generated and processed using the Canny edge detection algorithm. The edge direction histogram is quantized into 18 bins of 20 degrees each, thus a total of 18 edge features are extracted. 
All of these features are combined into a feature vector, which results in a vector with 36 values, we then normalize each feature to a normal distribution to eliminate the effect of different scales. The distance between pairs of images is computed as the Euclidean distance.

\subsection{Comparative performance evaluation}

We performed a series of experiments to show the effectiveness of the proposed method and compare its performance with with three state-of-the-art SVM feedback methods: SVM Active Learning [17], SVM Batch Mode Active Learning [20] and Dynamic Batch Sampling Mode [21]. To illustrate the actual situation of online users, randomly selected 20 images from the database are used to query, thus there will be 1600 query sessions. In the first step of each query session, the images in the database are ranked according to their Euclidean distances to the query. User's relevance adjustments are simulated automatically in each loop, and top 15 images are used to label related or unrelated. The images in the same class are considered relevant and the rest are considered irrelevant. All images are labeled in the feedback loop that will be used for learning system.

The retrieval results using the proposed algorithm without the relevance feedback are show in Fig. 2 a). The image at the top of left-hand corner is the query image, the images are framed in red is relevance to query image, the rest is non-relevance to query image. It's easy to realize that the number of relevance images to the query image is very limited; there are so many images though the distance is very close to the query image but very different semantics and vice versa. However, after four feedback loops, the number of relevant images of the proposed method has significantly improved as shown in Fig.2 b)

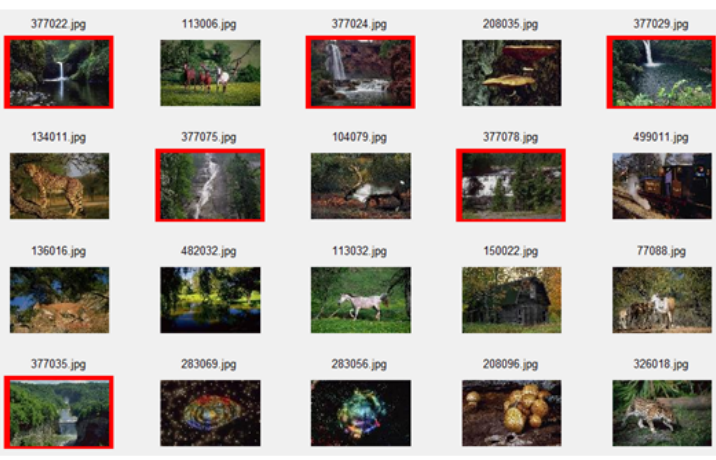

a)

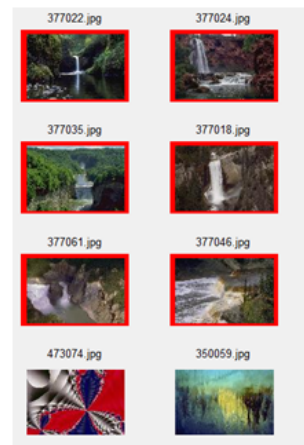

Figure 2. The retrieval results using the proposed algorithm: (a) the result without the relevance feedback, (b) the result after four feedback iterations

We use the Average Precision (AP) measure as an evaluation measure, which defined by NISTTREC video (TRECVID). The AP value that can be obtained at each iteration is defined as the average of precision value obtained after each relevant picture is retrieved. The precision value is the ratio between the retrieved relevant pictures and the number of pictures currently retrieved. In fact, using the result for only one query is not reliable. In order to evaluate the performance of CBIR, we need to compute the retrieval results for various image examples, then use the average values of their results. Moreover, by varying $N$, the number of returned images, we can plot Mean Average Precision as a function of $N$ with the number of result images fixed to 20 , $40,60,80$ and 100 . This experiment is to evaluate the efficient performance of all four methods in each case of user's requirement.

Several observations can be drawn from the results in Fig.3, Fig.4. First, we observe that retrieval performance of all the methods is improved after a number of rounds. This result indicates the important of RF technique in CBIR system. Second, we observe that our proposed method tends to be more effective than the others in early iterations. That is expected because SVM performance is low when the number of training examples for classification is small; and ranking images mainly based on the similarity measure of low-level features is better. However, as the number of the feedback iteration increases, the number of training examples seems to be large enough to learn a good SVM, so the similarity measure is no longer necessary. These results again show the effective of proposed for selecting a batch of informative unlabeled examples for relevance feedback in CBIR. 

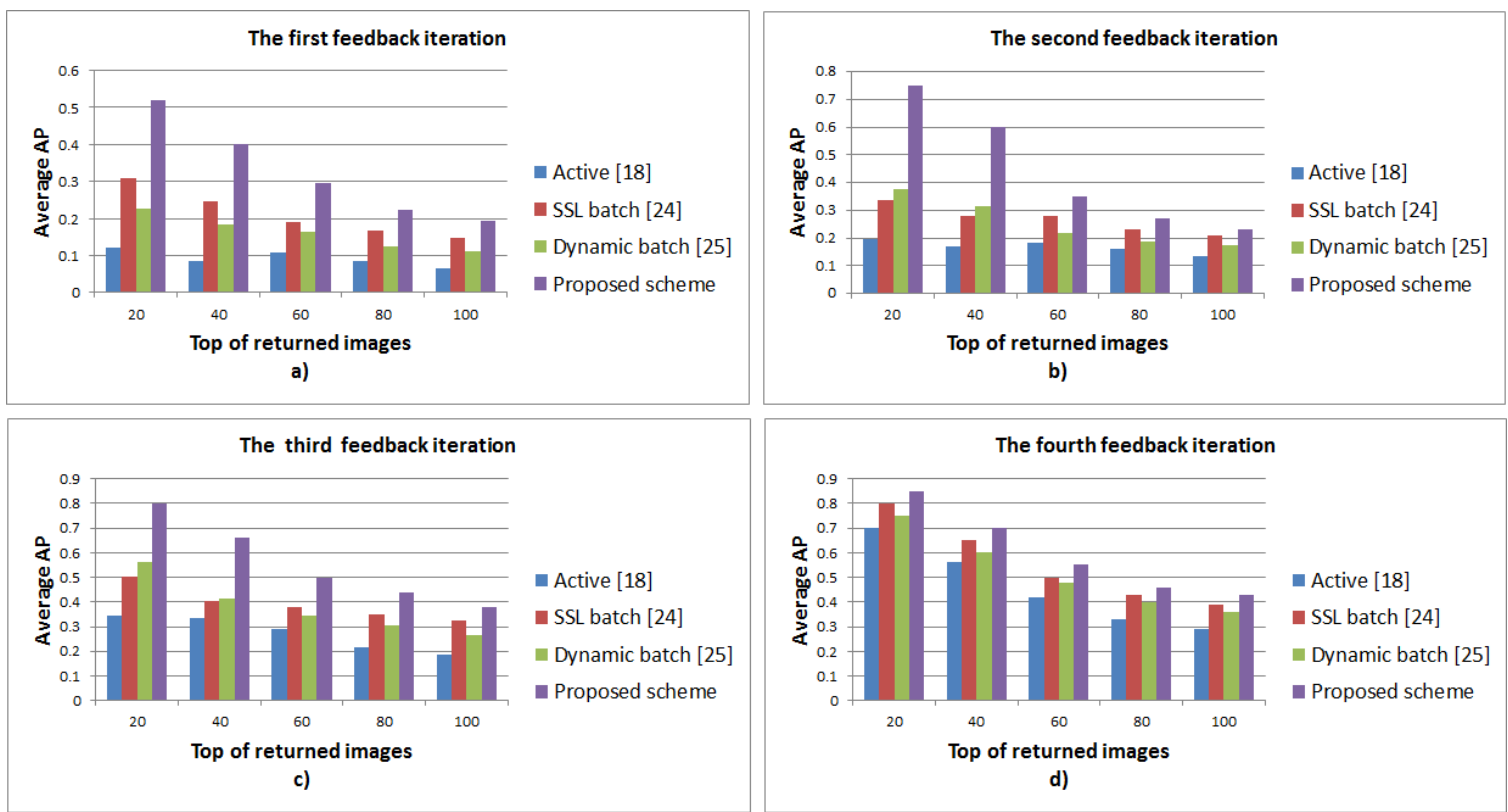

Figure 3. Relationship between average AP and number of returned images: (a) the first feedback iteration, (b) the second feedback iteration, (c) the third feedback iteration, and (d) the fourth feedback iteration.

\section{CONCLUSION}

In this paper, we have proposed a novel batch mode SVM active learning scheme for relevance feedback in CBIR. We choose a batch of feedback examples for the user to label using the combined ranking function instead of the SVM decision function used in traditional methods. Concretely, we combine two scores of SVM function and similarity measure to form a unique ranking function. With the help of combined ranking function, not only the adverse effect of inaccurate decision boundary due to lack of initially labelled samples can effectively be reduced, the retrieval performance can be further enhanced when there is sufficient number of initially labelled samples. The experimental results on a subset of COREL demonstrate the improvement by proposed scheme over the traditional schemes, especially when the number of initially labelled samples is small. As future developments of this work, we plan to extend the experimental on other datasets.

\section{ACKNOWLEDGEMENT}

This paper was supported in part by the Vietnam National Foundation for Science and Technology Development under NAFOSTED Grant 102.02.16.09 and Institute of Information Technology, VAST under Grant CS'14.3.

\section{REFERENCES}

[1] M. S. Lew, N. Sebe, C. Djeraba, and R. Jain, "Content-based multimedia information retrieval: State of the art and challenges," ACM Trans. Multimedia Comput. Commun. Appl., vol. 2, no. 1, pp. 1-19, Feb. 2006.

[2] Y. Liu, D. Zhang, G. Lu, and W.-Y. Ma, "A survey of content-based image retrieval with high-level semantics," Pattern Recognition, vol. 40, no. 1, pp. 262-282.

[3] R. Datta, D. Joshi, J. Li, and J. Z. Wang, "Image retrieval: Ideas, influences, and trends of the new age," ACM Computing Surveys, vol. 40, no. 2, pp. 1-60, May 2008.

[4] H. Bay, A. Ess, T. Tuytelaars, and L. V. Gool, "Speeded-up robust features (surf)," Computer Vision and Image Understanding, vol. 110, no. 3, pp. 346 - 359, 2008, similarity Matching in Computer Vision and Multimedia.

[5] J. Wu and J. M. Rehg, "Centrist: A visual descriptor for scene categorization," IEEE Transactions on Pattern Analysis and Machine Intelligence, vol. 33, no. 8, pp. 1489-1501, Aug 2011. 

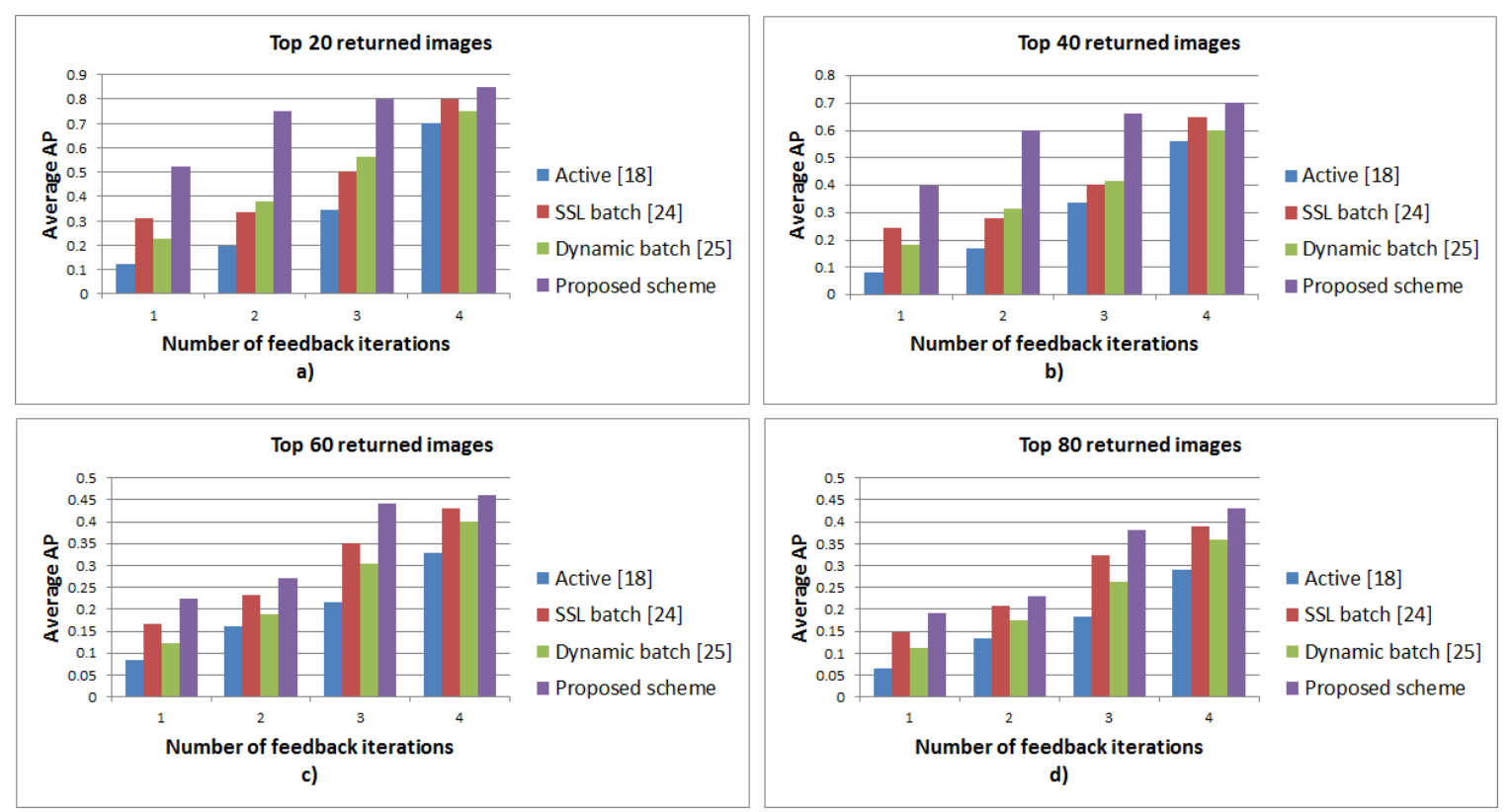

Figure 4. Relationship between average AP and number of iterations: (a) the top 20 returned images, (b) the top 40 returned images, (c) the top 60 returned images, and (d) the top 80 returned images

[6] L. Wu and S. C. H. Hoi, "Enhancing bag-of-words models with semantics-preserving metric learning," IEEE MultiMedia, vol. 18, no. 1, pp. 24-37, Jan 2011.

[7] N. T. Giang, N. Q. Tao, N. D. Dung, and N. T. The, "Skeleton based shape matching using reweighted random walks," in The proceding of the IEEE on 9th International Conference on Information, Communications and Signal Processing (ICICS), December 2013, pp. 1-5.

[8] Y. K. J. K. Zukuan WEI, Hongyeon KIM, “An efficient content based image retrieval scheme," TELKOMNIKA Indonesian Journal of Electrical Engineering, vol. 11, no. 11, p. 6986 6991, November 2013.

[9] O. M. A. B. Chawki Youness, El Asnaoui Khalid, "New method of content based image retrieval based on 2-d esprit method and the gabor filters," TELKOMNIKA Indonesian Journal of Electrical Engineering, vol. 15 , no. 2, pp. 313-320, August 2015.

[10] M. O. Y. Rui, T. S. Huang and S. Mehrotra, "Relevance feedback: A powerful tool for interactive contentbased image retrieval," IEEE Transactions on Circuits and Systems for Video Technology, vol. 8, pp. 644$655,1998$.

[11] B. Thomee and M. Lew, "Interactive search in image retrieval: a survey," International Journal of Multimedia Information Retrieval, vol. 1, no. 2, pp. 71-86, 2012.

[12] M. M. Rahman, P. Bhattacharya, and B. C. Desai, "A framework for medical image retrieval using machine learning and statistical similarity matching techniques with relevance feedback," IEEE Transactions on Information Technology in Biomedicine, vol. 11, no. 1, pp. 58-69, Jan. 2007.

[13] R. Min and H. Cheng, "Effective image retrieval using dominant color descriptor and fuzzy support vector machine," Pattern Recognition, vol. 42, no. 1, pp. 147 - 157, 2009.

[14] R.-S. Wu and W.-H. Chung, "Ensemble one-class support vector machines for content-based image retrieval," Expert Systems with Applications, vol. 36, no. 3, Part 1, pp. 4451 - 4459, 2009.

[15] X.-Y. Wang, J.-W. Chen, and H.-Y. Yang, "A new integrated svm classifiers for relevance feedback content-based image retrieval using em parameter estimation," Applied Soft Computing, vol. 11, no. 2, pp. $2787-2804,2011$.

[16] G. Li, "Improving relevance feedback in image retrieval by incorporating unlabelled images," TELKOMNIKA Indonesian Journal of Electrical Engineering, vol. 11, no. 7, pp. 3634-3640, 2013.

[17] S. Tong and E. Chang, "Support vector machine active learning for image retrieval," in Proceedings of the 10th ACM International Conference on Multimedia, 2001, pp. 107-118.

[18] S. C. H. Hoi and M. R. Lyu, "A semi-supervised active learning framework for image retrieval," in Proceedings of the 2005 IEEE Computer Society Conference on Computer Vision and Pattern Recognition, 
year $=2005$, pages $=302-309$.

[19] R. Liu, Y. Wang, T. Baba, D. Masumoto, and S. Nagata, "Svm-based active feedback in image retrieval using clustering and unlabeled data," Pattern Recognition, vol. 41, no. 8, pp. 2645 - 2655, 2008.

[20] S. C. H. Hoi, R. Jin, J. Zhu, and M. R. Lyu, "Semisupervised svm batch mode active learning with applications to image retrieval," Journal ACM Transactions on Information Systems, vol. 27, no. 3, pp. 16:1-16:29, May 2009.

[21] X. Zhang, J. Cheng, C. Xu, H. Lu, and S. Ma, "A dynamic batch sampling mode for svm active learning in image retrieval," in Recent Advances in Computer Science and Information Engineering, ser. Lecture Notes in Electrical Engineering, 2012, vol. 128, pp. 399-406.

[22] V. N. Vapnik, The Nature of Statistical Learning Theory. New York, NY, USA: Springer-Verlag New York, Inc., 1995. 